\title{
Effects of CCN3 on fibroblast proliferation, apoptosis and extracellular matrix production
}

\author{
ZHANPING REN $^{1}$, YUXIA HOU ${ }^{2}$, SIWEI MA ${ }^{1}$, YONGWEI TAO ${ }^{1}$, \\ JINFENG LI ${ }^{1}$, HUIQIN CAO ${ }^{1}$ and LINGLING JI ${ }^{2}$ \\ Departments of ${ }^{1}$ Cranio-Maxillofacial Trauma Plastic Surgery, and ${ }^{2}$ Orthodontics, \\ Stomatology Hospital of Xi'an Jiaotong University College of Medicine, Xi'an, Shaanxi 710004, P.R. China
}

Received January 11, 2014; Accepted March 14, 2014

DOI: 10.3892/ijmm.2014.1735

\begin{abstract}
CCN2 and CCN3 belong to the CCN family of proteins, which show a high level of structural similarity. Previous studies have shown that CCN2 mediates the ability of transforming growth factor (TGF) $-\beta$ to stimulate collagen synthesis, leading to keloid formation. CCN2 and CCN3 are opposing factors in regulating the promoter activity and secretion of this extracellular matrix (ECM) protein. Thus, we hypothesize that $\mathrm{CCN} 3$ possesses an anti-scarring effect. However, the exact mechanism of $\mathrm{CCN} 3$ in this anti-scarring effect remains unclear. The aim of this study was to investigate the mechanism of $\mathrm{CCN} 3$ in reducing scar formation. Palatal fibroblasts were obtained from the explants of the oral palatal mucosa of 8-week-old male Sprague-Dawley rats. CCN3 overexpression vector was constructed and then transfected into cells. The inhibitory effects of CCN3 on cell growth were detected via the 3-(4,5-dimethylthiazol-2-yl)-2,5-diphenyltetrazolium bromide (MTT) assay. Apoptosis was measured using an Annexin V-fluorescein isothiocyanate (FITC)/propidium iodide (PI) apoptosis detection kit and flow cytometry. The expression levels of collagen I, collagen III and $\alpha$-smooth muscle actin ( $\alpha$-SMA) were determined by western blot analysis and RT-PCR. Following treatment with TGF- $\beta 1$, we detected the expression of CCN3 and Smad1 in the fibroblasts. CCN3 significantly inhibited the growth and induction of apoptosis of fibroblasts. The expression of collagen I, collagen III and $\alpha$-SMA was lower in the CCN3-transfected group as compared to the control and vector groups. TGF- $\beta 1$ stimulation efficiently suppressed the expression of CCN3 at
\end{abstract}

Correspondence to: Dr Zhanping Ren, Department of CranioMaxillofacial Trauma Plastic Surgery, Stomatology Hospital of Xi'an Jiaotong University College of Medicine, No. 98 West Fifth Road, Xi'an, Shaanxi 710004, P.R. China

E-mail: zhanping_ren@126.com

Key words: CCN3, fibroblast, proliferation, apoptosis, extracellular matrix, $\alpha$-smooth muscle actin, transforming growth factor- $\beta 1 / \mathrm{Smad}$ the mRNA and protein levels, and CCN3 was required for TGF- $\beta 1$-induced Smad1 phosphorylation. Results of this study demonstrated that CCN3 is involved in the proliferation and apoptosis of fibroblasts and the synthesis of ECM proteins. Therefore, CCN3 may play an important role in the development of scar tissue, and may represent a novel therapeutic target for reducing scar formation.

\section{Introduction}

Orofacial clefts (OFCs), primarily cleft lip and cleft palate, are among the most common congenital anomalies in individuals in China and worldwide (1). The etiology of clefts in humans is a complex interaction between genetic and environmental factors (2). Nonetheless, our knowledge of their etiology and pathogenesis remains scarce, limiting our efforts at prevention (3). In many cases, the underlying molecular and cellular mechanisms that result in these debilitating anomalies remain largely unknown (4). OFCs lead to many complications including speech, hearing and feeding problems that require a team of maxillofacial and oral surgeons, head and neck surgeons, speech therapists and phoniatricians for treatment (2). Although surgical treatment has improved over the centuries, facial scarring remains a problem. Facial scars lead to cosmetic problems as well as to psychological effects such as poor self-esteem, emotional debilitation, embarrassment, and social isolation (5). Therefore, identifying ways to reduce scar formation is of utmost significance.

Scars are formed via the transformation of fibroblasts into myofibroblasts, accompanied by the proliferation of fibroblasts and excessive deposition of extracellular matrix (ECM) (6). Fibroblasts derived from surgical scar tissue produce high levels of $\alpha$-smooth muscle actin ( $\alpha$-SMA) and transforming growth factor (TGF)- $\beta 1$ (7). TGF- $\beta 1$ is one of the key cytokines in scar formation, and can act at different levels to increase collagen deposition. TGF- $\beta 1$ promotes fibroblast transformation into myofibroblasts, induces the synthesis of glycoproteins and matrix proteins, and inhibits collagen degradation by the reduction of metalloproteases and induction of protease inhibitors (8). Various anti-TGF- $\beta$ approaches have been successfully used to prevent fibrotic disorders of the skin and to reduce scar formation. 
The CCN protein family comprises the members: CCN1/CYR61, CCN2/CTGF, CCN3/NOV, CCN4/WISP1, CCN5/WISP2 and CCN6/WISP3. These proteins are induced by growth factors and cytokines such as TGF- $\beta$ and endothelin-1, and affect connective tissues, resulting in fibrosis and scarring (9). CCN2 is a member of the CCN family, which plays an important role in a variety of cell processes including angiogenesis, chondrogenesis and wound healing $(10,11)$. Findings of previous studies provided strong evidence that CCN2 plays a role in scars, particularly as a co-factor of TGF- $\beta 1$ (12). In addition, $C C N 2$ is likely to mediate the ability of TGF- $\beta 1$ to stimulate ECM synthesis and is a moderate inducer of collagen synthesis $(11,13)$. CCN2 and CCN3 are opposing factors in regulating collagen promoter activity and the secretion of this ECM protein. Riser et al demonstrated that $\mathrm{CCN} 3$, an endogenous regulator of pro-fibrotic changes, markedly downregulates collagen production and deposition (14). Collectively, these data suggest that CCN3 is a potentially important regulatory molecule in the TGF- $\beta 1 /$ Smad signaling pathway and a target for treatment in scar formation.

\section{Materials and methods}

Antibodies and reagents. Mouse anti-collagen I monoclonal antibody, mouse anti-collagen III monoclonal antibody, mouse anti- $\alpha$-SMA monoclonal antibody, mouse anti-Smad1 monoclonal antibody, mouse anti-Bcl-2 monoclonal antibody, mouse anti-Bax monoclonal antibody, mouse anti-CCN3 monoclonal antibody, mouse anti- $\beta$-actin monoclonal antibody and HRP-conjugated goat anti-mouse IgG were obtained from Abcam (Cambridge, UK). Dimethylsulfoxide (DMSO), propidium iodide (PI) and 3-(4,5-dimethylthiazol-2-yl)-2,5-diphenyltetrazolium bromide (MTT) assay were purchased from Bio-Rad (Hercules, CA, USA). The enhanced chemiluminescence western blot analysis detection reagents were obtained from Pierce Biotechnology, Inc. (Rockford, IL, USA). The fluorescein isothiocyanate (FITC)-Annexin V/PI apoptosis assay kit was purchased from Abcam.

Cell culture. Palatal fibroblasts were obtained from the explants of the oral palatal mucosa of 8-week-old male Sprague-Dawley rats (Shanghai Laboratory Animal Center, the Chinese Academy of Sciences), as reported previously (15), with minor modifications. Cells were cultured at $37^{\circ} \mathrm{C}$ under a humidified atmosphere of $5 \% \mathrm{CO}_{2}$ and $95 \%$ air and were grown in Dulbecco's modified Eagle's medium (DMEM; Gibco-BRL, Gaithersburg, MD, USA) supplemented with $10 \%$ heat-inactivated fetal bovine serum (CSL Ltd., Melbourne, Australia), $2 \mathrm{mM}$ L-glutamine, $50 \mathrm{mg} / \mathrm{ml}$ streptomycin and $50 \mathrm{U} / \mathrm{ml}$ penicillin (Gibco-BRL). The medium was changed every 2-3 days, and the cells were passaged with trypsin-EDTA (Gibco-BRL) when they became confluent. Experiments were performed using early passage cells (passage 5-10) derived from at least three independent animals without freezing.

Construction of CCN3 adenoviral vectors. Recombinant adenovirus was constructed as previously described (16). Briefly, the full length of CCN3 cDNA was amplified and subcloned into pAdTrack-CMV, an adenoviral shuttle plasmid.
A recombinant adenovirus expressing green fluorescent protein (GFP) was also used to assess infection efficiency. Then, the recombinant shuttle plasmids pAdTrack-CMV and pAdEasy-1 were homologously recombined in the Escherichia coli strain BJ5183. After sequencing, recombinant adenoviruses were packaged and produced in 293A cells.

Determination of cell proliferation. Cell proliferation was determined via the MTT assay and cell counting, as described previously with minor modifications (17). Briefly, $2 \times 10^{3}$ cells/well (3-wells per condition) were seeded in 96-well plates, and the cells were transfected with $\mathrm{CCN} 3$ adenoviral or blank vector, using Lipofectamine 2000 according to the manufacturer's instructions (Invitrogen Life Technologies, Grand Island, NY, USA). Culture medium was replaced every 2 days to avoid mitogen deprivation. At the indicated times, $10 \mu 1$ of an MTT solution $(5 \mathrm{mg} / \mathrm{ml}$ in phosphate-buffered solution) was added to the wells and cells were incubated for $4 \mathrm{~h}$ at $37^{\circ} \mathrm{C}, 5 \% \mathrm{CO}_{2}$. Following incubation, the culture medium in each well was replaced with $150 \mu \mathrm{l}$ of DMSO, and the plates were agitated to dissolve the dark blue crystals (formazan). Absorbance was measured at $570 \mathrm{~nm}$ using an enzyme-linked immunosorbent assay plate reader (Roche Diagnostics $\mathrm{GmbH}$, Penzberg,Germany). Cell proliferation was expressed as the percentage relative to the control. Each concentration was analyzed in triplicate and the experiment was repeated three times. All the experiments were performed at least three times and the results were averaged.

Analysis of cell apoptosis. The rate of apoptosis was measured via Annexin V-FITC and PI staining, followed by flow cytometry (Beckman Coulter, Inc., Brea, CA, USA). Briefly, the cells were seeded in a 6 -well plate at a density of $2 \times 10^{4}$ cells per well. After the cells reached $70 \%$ confluency, they were transfected with $\mathrm{CCN} 3$ adenoviral or blank vector. The cells were then trypsinized and suspended in $500 \mu \mathrm{l}$ of binding buffer containing $5 \mu \mathrm{l}$ Annexin V-FITC and $5 \mu \mathrm{l}$ PI (Abcam, Cambridge, MA, USA). After incubation in the dark for $1 \mathrm{~h}$, the cells were subjected to flow cytometry and the rate of cell apoptosis was determined.

$R T-P C R$.qRT-PCR was performed as previously described (18). Total-RNA was isolated using the RNeasy Plus Micro kit (Qiagen, Hilden, Germany) according to the manufacturer's instructions. Reverse transcription was performed using the High Capacity cDNA kit (Pierce Biotechnology, Inc.) according to the manufacturer's instructions. Real-time PCR was performed using the 7900HT Fast Real-Time PCR System (Bio-Rad) and was carried out using the SYBR Premix Ex Taq kit [Takara Biotechnology Co., Ltd., Dalian, China] at a final volume of $20 \mu \mathrm{l}$. Real-time PCRs were performed in triplicate. The expression levels of the relative genes were calculated using the $2^{-\Delta \Delta C T}$ method and the housekeeping gene $\beta$-actin was utilized as a control. The primer sets used were: collagenIforward,5'-TCCCCAGCCACAAAGAGTCTACA-3'; and reverse, 5'-GTGATTGGGTGGGATGTCTTCGTC-3'; collagen III forward, 5'-CTGCCATCCTGAACTCAA GAGTGG-3'; and reverse, 5'-CCATCCTCCAGAACTGTG TAGG-3'; TGF- $\beta 1$ forward, 5'-CAACAATTCCTGGCG ATACCTCA-3'; and reverse, 5'-GGTAGTGAACCCGTTGAT 


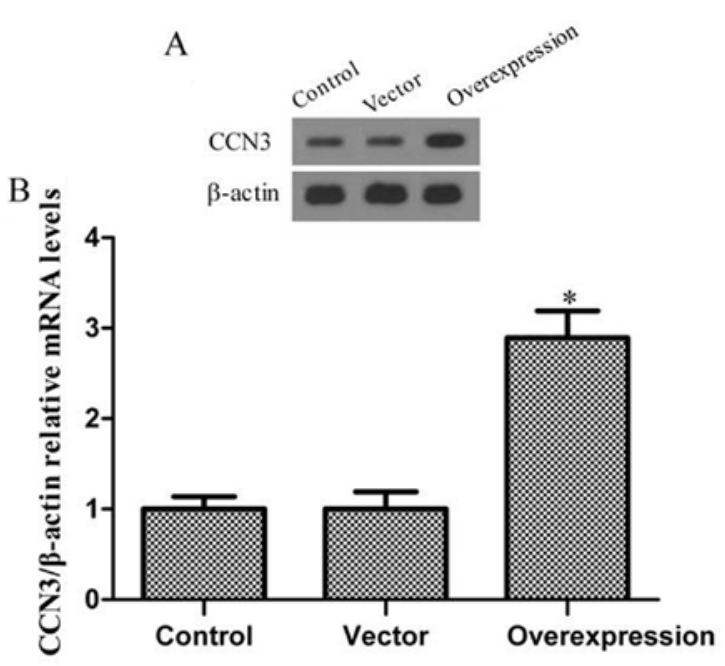

Figure 1. Effects of CCN3 transfection $(48 \mathrm{~h})$ on the expression levels of CCN3 in fibroblasts was determined by western blot analysis and real-time RT-PCR. (A) Western blot analysis of the protein levels of CCN3 in fibroblasts, $\beta$-actin was used as a loading control. (B) $\mathrm{CCN} 3 \beta$-actin relative mRNA expression levels \pm SD as detected by RT-PCR. Experiments were repeated at least three times. ${ }^{*} \mathrm{P}<0.05$ vs. control.

GTCCA-3' (19); $\alpha$-SMA forward, 5'-GACAATGGCTCT GGGCTCTGTAA-3'; and reverse, 5'-TGTGCTTCGTCACCC ACGTA-3' (19); Smad1 forward, 5'-ATGGACACGAACATG ACGAA-3'; and reverse, 5'-GCACCAGTGTTTTGGTT CCT-3' (20).

Western blot analysis. Total cellular proteins were extracted by incubating cells in lysis buffer according to the operating protocols. The protein concentrations in the cell lysates were determined using abicinchoninic acid assay protein assay kit (Hitachi, Tokyo, Japan). The same amount of protein from each sample was separated by sodium SDS-PAGE on a $12 \%$ agarose gel and electrophoretically transferred to a nitrocellulose membrane (Olympus, Tokyo, Japan). Mouse monoclonal anti-collagen I, anti-collagen III, anti-TGF- $\beta 1$, anti- $\alpha$-SMA, anti-CCN3, anti-Bcl-2, anti-Bax and anti-Smad1 were used as the primary antibody and anti-mouse IgG monoclonal antibody conjugated with horseradish peroxidase was used as the secondary antibody. Protein bands were detected using the West Femto system (Pierce Biotechnology, Inc.). Bands were visualized using ECL and semi-quantitative analysis was performed using $\beta$-actin as a protein loading control (21).

Statistical analysis. Experiments were carried out at least in triplicate and the results were expressed as mean \pm SD. Statistical analysis was performed using the SPSS 16.0 (SPSS, Inc., Chicago, IL, USA). Pairs of samples were compared using the Student's t-test. $\mathrm{P}<0.05$ was considered to indicate statistical significance.

\section{Results}

Determination of transfection effects. To test the efficiency of CCN3 transfection, western blot analysis and RT-PCR were employed to determine the expression level of the protein

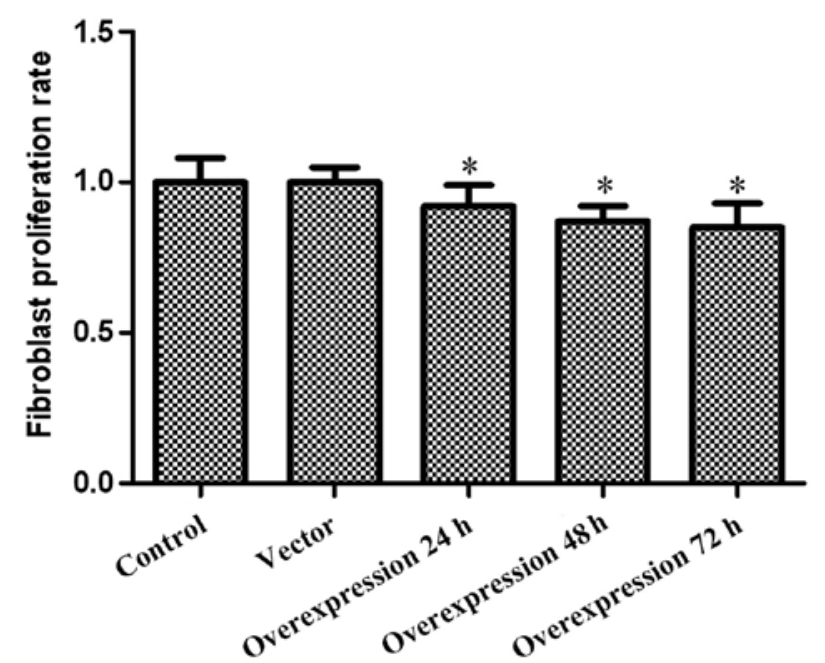

Figure 2. The effect of $\mathrm{CCN} 3$ on fibroblast proliferation. Fibroblast proliferation rate \pm SD of CCN3 as determined via 3-(4,5-dimethylthiazol-2-yl)-2,5-diphenyltetrazolium bromide (MTT) assay every $24 \mathrm{~h}$ after transfection, for up to $72 \mathrm{~h}$. Experiments were repeated at least three times. ${ }^{*} \mathrm{P}<0.05$ vs. control.

and mRNA. Fig. 1 shows that the fibroblast expressed only low levels of CCN3 in the control group. Moreover, transfection of the cells with adenoviral vector without CCN3 barely increased $\mathrm{CCN} 3$ expression. Of note, $\mathrm{CCN} 3$ expression significantly increased following transfection of adenoviral vector with $\mathrm{CCN} 3$ into fibroblasts. Collectively, these results indicate that transfection with $\mathrm{CCN} 3$-adenoviral vector effectively increased $\mathrm{CCN} 3$ expression in fibroblasts.

Effect of CCN3 on fibroblast proliferation. The impact of $\mathrm{CCN} 3$ on fibroblast proliferation was determined via MTT assay every $24 \mathrm{~h}$ after transfection, for up to $72 \mathrm{~h}$. The obtained results revealed that cell viability was, to a certain extent, inhibited by $\mathrm{CCN} 3$ in a time-dependent manner.As shown in Fig. 2, the CCN3-transfected group grew more slowly than the control groups. These results demonstrated that the overexpression of CCN3 inhibited fibroblast proliferation.

Effect of CCN3 on fibroblast apoptosis. Fibroblast apoptosis was detected via PI staining and the Annexin V method after $48 \mathrm{~h}$ of CCN3 transfection, followed by flow cytometry. As shown in Fig. 3A, there was a low level (4.9 and 4.5\%) of fibroblast apoptosis in the control and vector groups, although the percentage of apoptosis significantly increased to $22 \%(\mathrm{P}<0.05)$ after $48 \mathrm{~h}$ of $\mathrm{CCN} 3$ transfection.

To investigate whether CCN3 induces apoptosis in fibroblasts, the possible molecular mechanisms of CCN3 associated with apoptosis were assessed. Thus, we measured the expression of Bcl-2 and Bax proteins in cells $48 \mathrm{~h}$ post-transfection (Fig. 3B). The results showed that the expression of Bcl-2 decreased and the expression of Bax was simultaneously upregulated in the CCN3-transfected group compared with the control and vector groups $(\mathrm{P}<0.05)$. The apoptotic rate was significantly higher because of the upregulation of pro-apoptotic genes. These data revealed that $\mathrm{CCN} 3$ plays a critical role in promoting fibroblast apoptosis. 


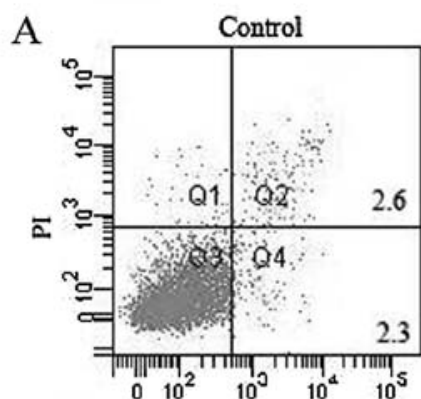

Annexin V-FITC

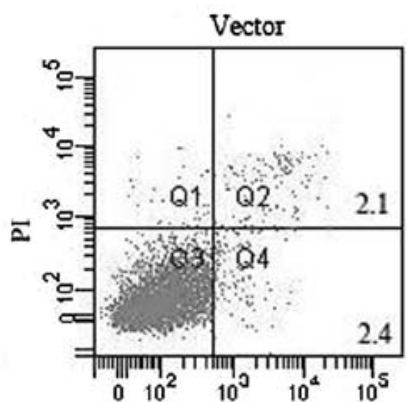

Annexin V-FITC

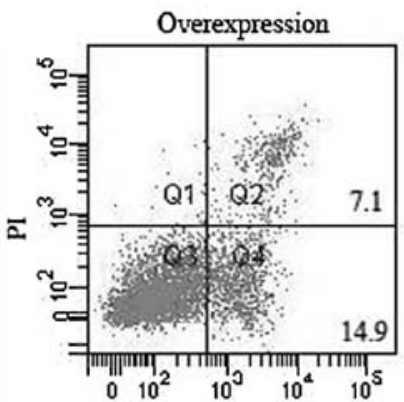

Annexin V-FITC

\section{B Control Vector Overexpression}

Bcl-2

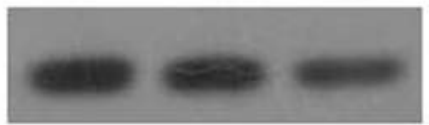

Bax

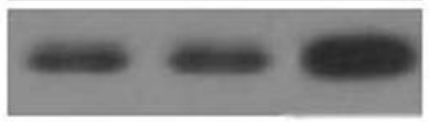

$\beta$-actin

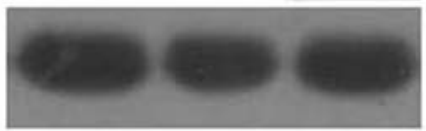

Figure 3. Effect of $\mathrm{CCN} 3$ on fibroblast apoptosis. (A) Fibroblast apoptosis was detected via propidium iodide (PI) staining and the Annexin $\mathrm{V}$ method after $48 \mathrm{~h}$ of CCN3 transfection, followed by flow cytometry. (B) CCN3 modulates the expression of Bcl-2 and Bax in fibroblasts. The expression of Bcl-2 and Bax were determined by western blot analysis. All experiments were repeated at least three times. All data are the means $\pm \operatorname{SD}(n=3)$.

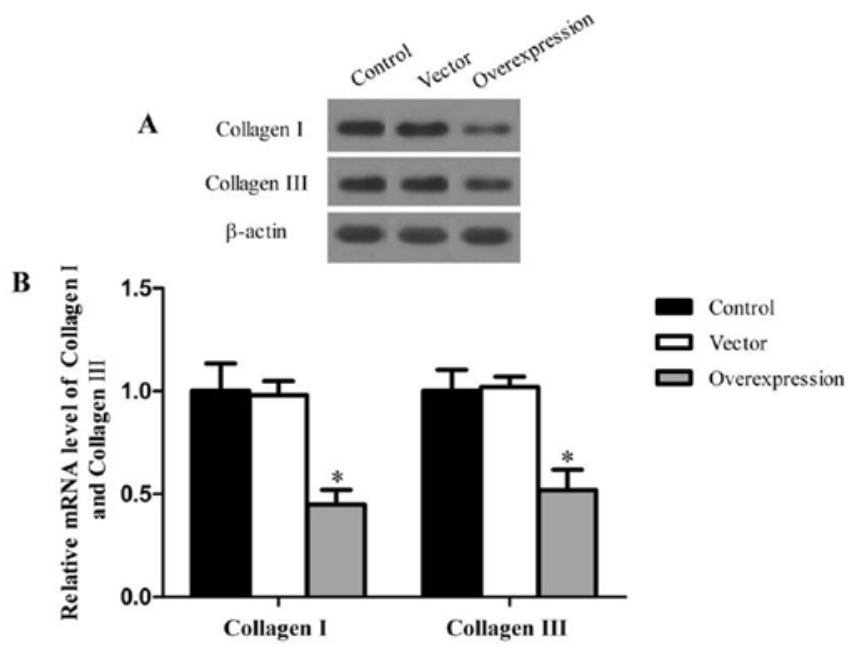

Figure 4. The effect of CCN3 on collagen I and collagen III. (A) The protein level of collagen I and collagen III were determined by western blot analysis, $\beta$-actin was used as a loading control. (B) Collagen I and collagen III mRNA expression level \pm SD as detected by RT-PCR. All experiments were repeated at least three times. ${ }^{*} \mathrm{P}<0.05$ vs. control.

Effect of CCN3 on collagen I and collagen III. In order to evaluate the effects of $\mathrm{CCN} 3$ on matrix production, we measured the protein and mRNA expression of collagen I and III, which constitute the bulk of the scar ECM. In RT-PCR and western blot analysis, the protein and mRNA levels of collagen I and III were significantly lower in the CCN3-transfected group compared with the control and vector groups $(\mathrm{P}<0.05)$ (Fig. 4).

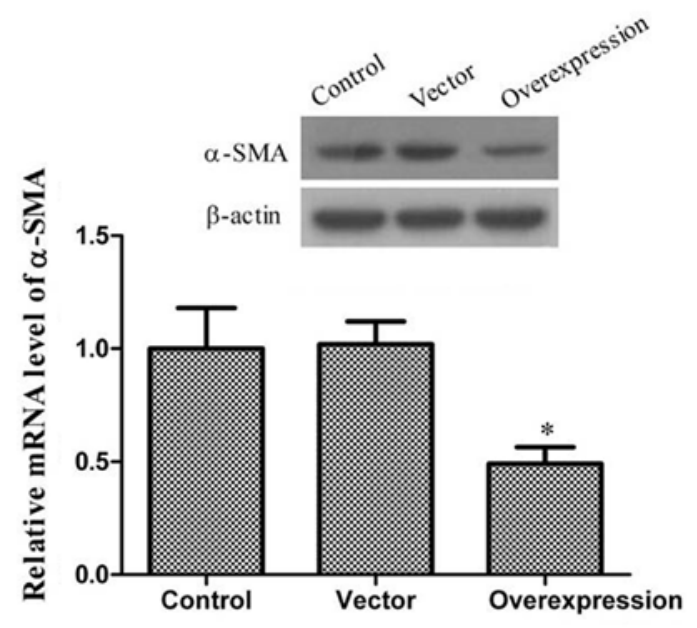

Figure 5. Effect of CCN3 on $\alpha$-smooth muscle actin ( $\alpha$-SMA). (A) The protein levels of $\alpha$-SMA was determined by western blot analysis, while $\beta$-actin was used as a loading control. (B) $\alpha$-SMA mRNA expression level \pm SD as detected by RT-PCR. Experiments were repeated at least three times. ${ }^{*} \mathrm{P}<0.05$ vs. control.

Effect of CCN3 on $\alpha$-SMA. $\alpha$-SMA, which plays a key role in the pathogenesis of scars, is the most widely used marker for myofibroblasts. The persistent presence of myofibroblasts is a distinctive feature of scars that contributes to excessive matrix production. As shown in Fig. 5, these results showed that the levels of $\alpha$-SMA expression were lower in the CCN3-transfected group as compared to the control and vector groups. 
A
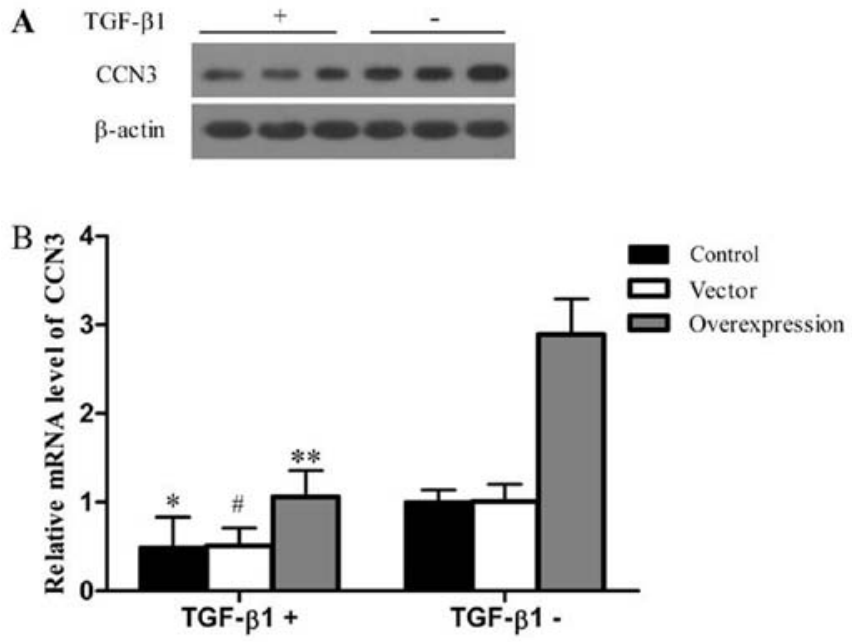

C TGF- $\beta 1$

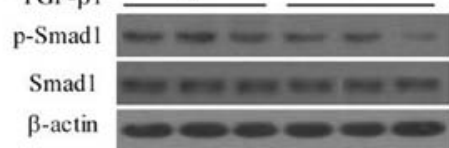

Figure 6. Effect of CCN3 on transforming growth factor (TGF)- $\beta 1 / \mathrm{Smad}$ signaling pathway. (A) Western blot analysis of the protein level of CCN3 in fibroblast with. (B) $\mathrm{CCN} 3 \mathrm{mRNA}$ expression level $\pm \mathrm{SD}$ in fibroblast as determined by RT-PCR analysis. (C) The phosphorylation and protein levels of Smad1 were determined by western blot analysis, while $\beta$-actin was used as a loading control. All experiments were repeated at least three times. ${ }^{*} \mathrm{P}<0.05$ vs. control.

CCN3 induces cell apoptosis and downregulates collagen I, collagen III and a-SMA via TGF- $\beta 1 /$ Smad signaling pathway. TGF- $\beta 1$ promotes fibroblast transformation into myofibroblasts and the synthesis of collagen (8). Therefore, we investigated the role of $\mathrm{CCN} 3$ in mediating the TGF- $\beta 1$-induced formation of collagen and $\alpha$-SMA. Under exogenous TGF- $\beta 1$ stimulation, we determined the protein and mRNA levels of CCN3. TGF- $\beta 1$ stimulation efficiently suppressed the expression of CCN3 at the mRNA and protein levels (Fig. 6A and B). Previously it was shown that the classical Smad1 pathway plays a role in the TGF- $\beta 1$ regulation of collagen and $\alpha$-SMA expression (11). We then determined the activation of Smad1 pathways in response to TGF- $\beta 1$ stimulation. As shown in Fig. 6C, the phosphorylation of Smad1 was significantly lower in the CCN3-transfected group compared with the control and vector groups. Furthermore, the phosphorylation of Smad1 in response to TGF- $\beta 1$ was higher than that without TGF- $\beta 1$ stimulation. However, there was no apparent change in the protein level of Smad1 following TGF- $\beta 1$ stimulation.

\section{Discussion}

To the best of our knowledge, this study has shown for the first time that $\mathrm{CCN} 3$ induces cell apoptosis and downregulates collagen I, collagen III and $\alpha$-SMA via the TGF- $\beta 1 / \mathrm{Smad}$ signaling pathway.

Treatment of scars following cleft lip and palate operations is problematic, with no single modality having uniformly satisfactory results. The techniques currently used to correct cleft lip cannot successfully solve the problem of scarring. Several effective therapeutic measures can be offered for the clinical treatment of exuberant scars; however, these therapies are far from satisfactory. Therefore, there is a need to find approaches that are suitable for the treatment and better control of scars.

Studies on the mechanism of scar formation have shown that TGF- $\beta 1$ is one of the most important elements in the process of scar formation (22). Previous studies have shown that CCN2 plays a crucial role in concert with TGF- $\beta 1$ during the formation and development of fibrotic disorders or scarring (18). $\mathrm{CCN} 2$ is known to mediate the ability of TGF- $\beta$ to stimulate ECM synthesis, leading to keloid formation (13). However, CCN3 may perform opposing functions that modulate the effects of CCN2. Thus, we hypothesize that CCN3 exerts an anti-scarring effect. However, the exact mechanism of involvement of CCN3 in scarring is poorly understood. This study aimed to address this issue.

In the present study, we evaluated the effects of the overexpression of $\mathrm{CCN} 3$ on the inhibition of proliferation and promotion of apoptosis in palatal fibroblasts, along with its mechanism of action. The results showed that the overexpression of CCN3 inhibited palatal fibroblast proliferation and induced cell apoptosis. Overexpression of $\mathrm{CCN} 3$ can dowregulate $\mathrm{Bcl}-2$ expression and upregulate Bax expression. It is likely that CCN3 induces palatal fibroblast apoptosis by inhibiting Bcl-2 expression and activating Bax.

Type I and III collagens are the major proteins comprising the ECM (23). However, excess deposition of ECM components, particularly collagen, can result in scarring (24). Therefore, we assessed the protein and mRNA expression of collagen I and III, and found that CCN3 downregulates the expression of the two proteins.

$\alpha$-SMA is a hallmark of myofibroblast differentiation, which plays a key role in the pathogenesis of scars (25). The persistent presence of myofibroblasts is a distinctive feature of scars that contributes to excessive matrix production. In our study, the downregulation of $\alpha$-SMA by CCN3 was considered one of the signals inhibiting fibroblast differentiation. These results suggest that $\mathrm{CCN} 3$ is crucial in scarring.

Moreover, results of this study suggest that $\mathrm{CCN} 3$ induces cell apoptosis and downregulates collagen I, collagen III and $\alpha$-SMA via the TGF- $\beta 1 /$ Smad signaling pathway. Under exogenous TGF- $\beta 1$ stimulation, CCN3 expression was suppressed and Smad1 phosphorylation increased. Thus, CCN3 induces cell apoptosis and downregulates collagen I, collagen III and $\alpha$-SMA via the TGF- $\beta 1 /$ Smad signaling pathway.

In summary, this study has demonstrated that $\mathrm{CCN} 3$ is involved in the proliferation and apoptosis of fibroblasts and the synthesis of ECM proteins. Therefore, CCN3 may play an important role in the development of scars, and may represent a novel therapeutic target for reducing scar formation.

\section{References}

1. Marazita ML: The evolution of human genetic studies of cleft lip and cleft palate. Annu Rev Genomics Hum Genet 13: 263-283, 2012.

2. Reiter R, Haase S and Brosch S: [Orofacial clefts]. Laryngorhinootologie 91: 84-95, 2012. 
3. Cox TC, Luquetti DV and Cunningham ML: Perspectives and challenges in advancing research into craniofacial anomalies. Am J Med Genet C Semin Med Genet 163: 213-217, 2013.

4. Metzis V, Courtney AD, Kerr MC, Ferguson C, Rondón Galeano GM, Parton RG, Wainwright BJ and Wicking C: Patched1 is required in neural crest cells for the prevention of orofacial clefts. Hum Mol Genet 22: 5026-5035, 2013.

5. Díaz Casado GH and Díaz Grávalos GJ: [Orofacial closure defects: cleft lip and palate. A literature review]. Semergen 39: 267-271, 2013.

6. Hoogewerf CJ, van Baar ME, Middelkoop E and van Loey NE: Patient reported facial scar assessment: directions for the professional. Burns 40: 347-353, 2014.

7. Goldberg MT, Han YP, Yan C, Shaw MC and Garner WL: TNF-alpha suppresses alpha-smooth muscle actin expression in human dermal fibroblasts: an implication for abnormal wound healing. J Invest Dermatol 127: 2645-2655, 2007.

8. Omori S, Kitagawa H, Koike J, Fujita H, Hida M, Pringle KC and Awazu M: Activated extracellular signal-regulated kinase correlates with cyst formation and transforming growth factor-beta expression in fetal obstructive uropathy. Kidney Int 73: 1031-1037, 2008.

9. Leask A and Abraham DJ: All in the CCN family: essential matricellular signaling modulators emerge from the bunker. J Cell Sci 119: 4803-4810, 2006.

10. Moussad EE and Brigstock DR: Connective tissue growth factor: what's in a name? Mol Genet Metab 71: 276-292, 2000.

11. Nakerakanti SS, Bujor AM and Trojanowska M: CCN2 is required for the TGF- $\beta$ induced activation of Smad1-Erk1/2 signaling network. PLoS One 6: e21911, 2011.

12. Hahn A, Heusinger-Ribeiro J, Lanz T, Zenkel $S$ and Goppelt-Struebe M: Induction of connective tissue growth factor by activation of heptahelical receptors. Modulation by Rho proteins and the actin cytoskeleton. J Biol Chem 275: 37429-37435, 2000.

13. Sisco M, Kryger ZB, O'Shaughnessy KD, Kim PS, Schultz GS, Ding XZ, Roy NK, Dean NM and Mustoe TA: Antisense inhibition of connective tissue growth factor (CTGF/CCN2) mRNA limits hypertrophic scarring without affecting wound healing in vivo. Wound Repair Regen 16: 661-673, 2008.

14. Riser BL, Bhagavathula N, Perone P, Garchow K, Xu Y, Fisher GJ, Najmabadi F, Attili D and Varani J: Gadolinium-induced fibrosis is counter-regulated by $\mathrm{CCN} 3$ in human dermal fibroblasts: a model for potential treatment of nephrogenic systemic fibrosis. J Cell Commun Signal 6: 97-105, 2012.
15. Kanda T, Funato N, Baba Y and Kuroda T: Evidence for fibroblast growth factor receptors in myofibroblasts during palatal mucoperiosteal repair. Arch Oral Biol 48: 213-221, 2003.

16. Liu L, Zhang G, Liang Z, Liu X, Li T, Fan J, Bai J and Wang Y: MicroRNA-15b enhances hypoxia/reoxygenation-induced apoptosis of cardiomyocytes via a mitochondrial apoptotic pathway. Apoptosis 19: 19-29, 2014.

17. Crowe J, Aubareda A, McNamee K, Przybycien PM, Lu X, Williams RO, Bou-Gharios G, Saklatvala J and Dean JL: Heat shock protein B1-deficient mice display impaired wound healing. PLoS One 8: e77383, 2013.

18. Hu X, Wang H, Liu J, Fang X, Tao K, Wang Y, Li N, Shi J, Wang Y, Ji P, Cai W, Bai X, Zhu X, Han J and Hu D: The role of ERK and JNK signaling in connective tissue growth factor induced extracellular matrix protein production and scar formation. Arch Dermatol Res 305: 433-445, 2013.

19. Yang G, Crawford RC and Wang JH: Proliferation and collagen production of human patellar tendon fibroblasts in response to cyclic uniaxial stretching in serum-free conditions. J Biomech 37: 1543-1550, 2004.

20. Fujiwara N, Doi T, Gosemann JH, Kutasy B, Friedmacher F and Puri P: Smad1 and WIF1 genes are downregulated during saccular stage of lung development in the nitrofen rat model. Pediatr Surg Int 28: 189-193, 2012.

21. Yuan J, Huang G, Xiao Z, Lin L and Han T: Overexpression of $\beta$-NGF promotes differentiation of bone marrow mesenchymal stem cells into neurons through regulation of AKT and MAPK pathway. Mol Cell Biochem 383: 201-211, 2013.

22. Cannata A, Petrella D, Russo CF, Bruschi G, Fratto P, Gambacorta M and Martinelli L: Postsurgical intrapericardial adhesions: mechanisms of formation and prevention. Ann Thorac Surg 95: 1818-1826, 2013.

23. Pickering JG: Regulation of vascular cell behavior by collagen: form is function. Circ Res 88: 458-459, 2001.

24. Sato M: Upregulation of the Wnt/beta-catenin pathway induced by transforming growth factor-beta in hypertrophic scars and keloids. Acta Derm Venereol 86: 300-307, 2006.

25. Abdalla M, Goc A, Segar L and Somanath PR: Akt1 mediates $\alpha$-smooth muscle actin expression and myofibroblast differentiation via myocardin and serum response factor. J Biol Chem 288: 33483-33493, 2013. 